EDITORIAI

\title{
New developments in personality and identity research across cultures
}

\author{
Byron G. Adams ${ }^{1,2}$, Radosveta Dimitrova ${ }^{3}$ \\ 1: Tilburg University, the Netherlands \\ 2: University of Johannesburg, South Africa \\ 3: Stockholm University, Sweden
}

$\mathrm{W}$

hile this journal mainly focuses on personality aspects in psychology, there is a close link between personality and identity (McAdams, 1995). Essentially an individual's personality is characterized mainly by her/his thoughts, emotions and behaviors, generally captured by traits (McCrae \& Costa, 2003), whereas the individual's identity entails defining oneself as both unique and similar to others, in relation to others and within the social context (Munday, 2006), making up the individual's life story (McAdams \& Olson, 2010). These aspects are mainly dealt with from a Western (Northern American and Western European) perspective, often with theoretical and methodological contributions from non-Western contexts (Arnett, 2008). It is clear that personality and identity have much in common and that both are crucial to our everyday psychological functioning. This issue examines personality, identity and their correlates (notably well-being) outside the Western-European and Northern American context where these are usually studied. The final article integrates the previous articles by proposing a conceptual model that integrates personality and identity.

We present methodological, empirical, and theoretical contributions and their relevance for aspects of personality and identity both within and across different national and cultural groups. These groups (e.g., Black and White South Africans, Turkish Bulgarians, and the Slovene minority in Italy) and nations (e.g., South Africa, Slovenia, and Croatia) are often understudied in both personality and identity literature. With the empirical work in non-Western groups and contexts, we first counter selection bias (i.e., the vast majority of research stemming from Western contexts; Peeters, de Jonge, \& Taris, 2014) often prevalent in personality and identity studies. While this issue highlights both the importance of methodological aspects (as in the first article) and theoretical perspectives (as with the final article) when considering personality and identity in the two conceptual articles, the empirical work addresses the validity of Western theoretical models on personality and identity, particularly as antecedents of well-being, in non-Western contexts. In the first (theoretical) article $\mathrm{He}$ and van de Vijver describe appropriate research methods for avoiding common pitfalls in studying personality across cultures. They focus on sampling issues, test adaptations, and the combination of emic and etic approaches.

In the second and third articles, the authors evaluate the importance of personality traits for well-being. In the second article, Nel et al. address the nomological network of the South African Personality Inventory (SAPI) across Black and White South African emerging adults. In this study, an earlier version of the SAPI, which comprises nine factors (Conscientiousness, Emotional Stability, Extraversion, Facilitating, Integrity, Intellect, Openness, Relationship Harmony and Soft-heartedness; Hill et al., 2013), highlights the adequacy of this empirically developed measure of personality for well-being of emerging adults across ethnocultural groups in South Africa. 
In the third article, Tatalović Vorkapić and Peloza evaluate the relationship between personality as traditionally measured by the Big Five (openness to experience, conscientiousness, agreeableness, extraversion, and emotional stability; McCrae \& Costa, 2003) with the life satisfaction, happiness, and optimism of pre-school and primary school teachers in Croatia. This article evaluates both differences and similarities in how personality informs well-being for each of these teaching groups within the Croatian context. As the final contribution related to personality, the fourth article, by Kozina, evaluates trait aggression in late childhood and early adolescents in Croatia in line with established theoretical perspectives. She considers specific subtypes of aggression deemed relevant for the Slovene context (i.e., general trait aggression and four factors: physical aggression, verbal aggression, inner aggression and aggression towards authority; Kozina, 2013) and provides implications for development of aggression within and across the two age groups.

In the next two articles we consider the importance of identity for well-being across minority and mainstream groups in Bulgaria and Italy. First, Dimitrova, Chasiotis, Bender and van de Vijver study youth from a Turkish-Bulgarian minority group, a Muslim-Bulgarian minority group, and a Mainstream group in Bulgaria. They are the first authors to report on the importance of ethnic, familial, and religious identity as well as the identity status model for well-being in this cultural context. Second, Kosic and Dimitrova evaluate the importance of collective identity (i.e., ethnic, national, familial, and religious identities) for well-being across youth from a Slovene minority group and an Italian mainstream group. Both studies provide valuable insight into how more holistic approaches for considering identity may be valuable for understanding the well-being of youth.

The final article in this thematic issue is an invited commentary by Syed on the preceding articles. Syed used the articles as inspiration for a broader discussion on personality and identity, highlighting the incoherence present in both this issue and in general on how the relationship between personality and identity is often approached. He explores the overlapping, yet distinct nature between personality and identity and describes a three-level model of personality (i.e., traits, characteristic adaptations, and the integrated life story) which is argued to encompass identity (McAdams \& Olson, 2010). In addition, he discusses this model in relation to research in cultural psychology, mixed methodological approaches, and understanding psychological outcomes.

In conclusion, as editors we aimed to provide readers with novel insights from underrepresented cultural groups and national (mono-/multicultural) contexts on identity and personality. In addition, the article by Syed highlights the gap which exists when considering these constructs and their relationship and a possible way to integrate personality and identity more. Finally, by describing the close correspondence in issues studied in the personality and identity literature, we aim to contribute to better coordination and ultimately the merging of the fields of personality and identity to provide a more holistic perspective of who people are.

\section{RefERENCES}

Arnett, J. J. (2008). The neglected 95\%: why American psychology needs to become less American. American Psychologist, 63, 602.

Hill, C., Nel, J. A., van de Vijver, F. J., Meiring, D., Valchev, V. H., Adams, B. G., \& De Bruin, G. P. (2013). Developing and testing items for the South African Personality Inventory (SAPI). SA Journal of Industrial Psychology, 39, 1-13.

Kozina, A. (2013). The LA Aggression Scale for Elementary School and Upper Secondary School Students: Examination of Psychometric Properties of a New Multidimensional Measure of Self-Reported Aggression. Psihologija, 46, 245-259.

McAdams, D. P. (1995). What do we know when we know a person? Journal of Personality, 63, 365-396.

McAdams, D. P., \& Olson, B. D. (2010). Personality development: Continuity and change over the life course. Annual Review of Psychology, 61, 517-542.

McCrae, R. R., \& Costa, P. T. (2003). Personality in adulthood: A five-factor theory perspective. New York, NY: Guilford Press.

Munday, J. (2006). Identity in focus the use of focus groups to study the construction of collective identity. Sociology, 40, 89-105.

Peeters, M., De Jonge, J., \& Taris, T. (2014). An introduction to contemporary work psychology. Chichester, West Sussex, UK: John Wiley \& Sons. 